\title{
Enhancing Learning through Simulation Activities
}

Lisa McDavid“, MSN, RN

Assistant Professor, Morehead State University, Center for Health, Education, and Research, USA

*Corresponding author: Lisa McDavid, Assistant Professor, Morehead State University, Center for Health, Education, and Research, 316 W. 2nd St., Suite 201, Morehead, KY. 40351, Tel: 606-783-2296; Fax: 606-783-9123; E-maill.mcdavid@moreheadstate.edu

Received date: June 12, 2014, Accepted date: September 27, 2014, Published date: September 30, 2014

Copyright: () 2014 McDavid L. This is an open-access article distributed under the terms of the Creative Commons Attribution License, which permits unrestricted use, distribution, and reproduction in any medium, provided the original author and source are credited.

\begin{abstract}
Simulation activities performed in nursing programs can provide many learning opportunities for nursing students. Real life simulation activities, allow students to put their knowledge into practice, while recognizing areas of weakness that need improvement. The environment to perform these activities is a safe learning environment to allow students to make and recognize mistakes prior to entering the career field. Debriefing activities allow for reflection to encourage students to self-evaluate and recognize areas for improvement. Faculty encourage students and assist in improving areas of weakness. By participating in the activities, student's cognitive and psychomotor skills can be enhanced and students can gain a better understanding of didactic material.
\end{abstract}

\section{Enhancing Learning through Simulation Activities}

Integrating simulation into nursing programs allows students many learning opportunities. Simulation gives the students a hands-on approach to perform skills learned throughout their nursing education. If students are given the opportunity to perform and learn in a safe environment, they may possibly be more comfortable in recognizing their areas of weakness and where improvement is greatly needed. The following article discusses how one Associate Degree Nursing program schedules and performs simulation activities to enhance learning of didactic content. During the activities, students perform learned skills, medication administration, and learn how to model professional nursing practices. Once the simulation has been completed, a debriefing session takes place for students and faculty to reflect, discuss and recognize areas of weakness that require improvement by the student.

\section{Literature Review}

Simulation has been increasing in nursing education throughout the last 40 years. The activities do not always include the use of lowfidelity or high-fidelity manikins, but may utilize other forms. Other forms of simulation include, role-play, games, or computer-assisted instruction (CAI) [1]. Any form of simulation can be beneficial to enhance cognitive and psychomotor development among nursing students. With the number of nursing students on the rise and a limited number of clinical sites available for students to practice, "simulation has become an integral part of nursing education" [1].

Simulation is beneficial in the nursing educational setting if applied appropriately. Depending on clinical site placement, students may not get exposure to certain critical medical issues. Getting students involved assists them to demonstrate their ability to apply knowledge and critically think. Simulation activities must be planned carefully by the instructor and include specific tasks and/or issues for students to carry out appropriate care to simulate a real life-life situation. Each task performed should have results to further allow students to critically think, which adds to the realism of the simulation [2]. A Professor of Nursing at Pasco Hernando Community College in
Florida and an Education Specialist at Florida Hospital Carrollwood in Florida implemented a pilot program in an Associate Degree Nursing Program, requiring students to develop their own simulation scenarios. A clinical group of students was divided into two smaller groups to create scenarios for a simulation. Once the scenarios were developed, each group ran their developed scenario with the other group. Students were responsible for developing all elements for the simulation, including diagnostic testing, laboratory values, physician orders, medications, and any other pertinent information needed for the scenario [3]. Requiring students to develop scenarios allows students to critically think and develop subjective and objective data that would typically be seen in a real life clinical setting. In conclusion of the study, student involvement in gathering all data and constructing a scenario was useful in facilitating "critical thinking and clinical judgment skills" [3].

Simulation activities can be beneficial in enhancing basic nursing skills to complex skills. A common simulation activity includes the learning and performing Basic Life Support (BLS) and Advanced Cardiac Life Support (ACLS). A study was conducted to test the effectiveness of simulation with students' ability and confidence to adequately perform necessary skills according to the BLS and ACLS guidelines. The experimental group participated in a simulated scenario along with a PowerPoint presentation, while the control group only attended the presentation. At the end of the study, findings demonstrated how simulation elicits critical thinking among nursing students and helps students achieve confidence to perform professional skills [4].

\section{Simulation Activities}

Throughout the Associate Degree Nursing Program (ADNP) at Morehead State University, students participate in simulation activities that relate to the content learned in the course. The simulated activities are carried out use high-fidelity manikins. The lab area is designed to simulate a real world nursing unit. A nursing station is setup and the clinical simulation specialist simulates the role of secretary during the simulation activities. Each lab contains nine beds specially equipped with a working controls and call-light system. Students have the 
opportunity to demonstrate the use of vital sign machines, the Omnicell for medication administration, Intravenous (IV) pumps, and a fully stocked emergency crash cart. Supplies are available to students in order to enable them to perform their nursing skills such as, IV insertion, Foley ${ }^{\oplus}$ catheter insertion, nasogastric tube (NGT) insertion, and dressing changes. Faculty believe it is important to create realistic scenarios in a realistic environment to prepare the students for eventual nurse practice in a professional setting.

Techniques for skills, especially sterile technique, are closely monitored by faculty to ensure appropriate technique. When students do not practice the skill correctly they are required to return to the lab during open lab times and remediate to improve this important procedure. The patient care laboratory coordinator and simulation specialist are available to assist students with their learning needs.

\section{Specialty Lab Experiences}

Faculty schedule simulation labs according to content being delivered in the didactic portion of the course to make simulation activities more beneficial. The faculty schedule two specific simulation labs to assist students in better understanding cardiac content, one simulation to enhance neurology content, and one comprehensive inter-professional simulation at the end of the semester. The course is the final semester of the Associate Degree Nursing Program with a focus on acute Medical Surgical issues. The simulation labs include:

\section{Advanced Cardiac Life Support (ACLS) Simulation Lab}

The Advanced Cardiac Life Support (ACLS) simulation lab is scheduled during the cardiac didactic portion of the course. During this simulation, students are encouraged to critically think during a life-threatening emergency situation. The simulation uses a highfidelity manikin that adequately displays a cardiac arrest event. Students work together in groups of 4-5 to recognize an emergency event and carry out the necessary procedures. This simulation activity allows students to demonstrate their knowledge of Basic Life Support (BLS), ACLS algorithms for specific cardiac rhythms, team work, prioritization and adequate communication. The fully stocked simulated crash cart gives students access to the many emergency medications needed for the event and the ability to defibrillate the patient to correct the cardiac arrhythmia. Students perform necessary skills in order to successfully complete the scenario and are encouraged to recognize areas of safety issues that can be harmful to the patient as well as the student.

\section{Cardiac Simulation Lab}

The Cardiac simulation lab is also scheduled during the cardiac didactic portion of the course; however the focus is more toward learning cardiac rhythms and identifying complications. The cardiac simulation lab corresponds with the affiliated clinic course in the final semester of the program. Students are expected to recognize cardiac arrhythmias such as tachycardia and bradycardia. Students use their ACLS algorithms to follow appropriate protocols to treat the rhythm being displayed. Students are evaluated on their ability to follow the algorithm correctly and administer the medications with the appropriate doses. Each arrhythmia is demonstrated in a separate brief scenario in order for the students to learn the appropriate steps to treat each specific rhythm.

The cardiac simulation lab also includes a myocardial infarction (MI) scenario. Students demonstrate their ability to recognize signs and symptoms of a MI and carry out treatment protocols. Students retrieve simulated lab values that demonstrate the effects of a MI and a simulated 12-lead Electrocardiogram (EKG) to display changes in the cardiac rhythm associated with a MI.

\section{Neurology Simulation Lab}

Students participate in scenarios to recognize bacterial meningitis, stroke, and seizure during the neurology didactic portion of the course. During each scenario, students receive simulated labs and administer simulated medications to appropriately treat the issue being demonstrated. During these scenarios students are exposed to protocols for treatment, isolation requirements and various safety measures. Specific safety measures include seizure precautions, such as; ensuring suctioning is at the bedside, padding the side rails, and having the bed in low position. In the stroke and seizure scenarios, students actively participate as the patient. Students are required to be prepared to adequately demonstrate the signs of symptoms of each condition. Allowing students to participate in this role helps the student to understand the situation from view of a patient.

\section{Inter-Professional Simulation}

At the end of the final semester, students participate in an interprofessional simulation. This particular simulation provides a comprehensive group of patients demonstrating several conditions that have been learned throughout the program. First year students are involved and participate in the role of the patient for each scenario. First year students are given scripts and assisted in adequately demonstrating the appropriate scenario. Students are placed into groups of four. One student takes on the role of charge nurse and the other three are assigned three simulation patients. Students are given 45 minutes to meet the objectives of each scenario they are assigned. For example, once scenario involves a patient who is recovering from a total knee replacement. The student objectives for this scenario is to recognize redness, swelling, and itching at the incision site. The student should also obtain the most recent complete blood count (CBC) and recognize an elevation in the white blood cells (WBC). Another scenario involves a patient recovering one day after a cardiac catheterization. For this scenario, students must notice bleeding from the catheterization femoral site, obtain the most recent partial thromboplastin time (PTT), stop the heparin drip that is infusing, and notify the physician.

During the simulation, students are or have been exposed to physicians or simulated physicians. The exposure has been face-to-face in the past and currently occurs via phone conversations. When actual physicians are unavailable, faculty simulate the role of the physician. Students simulate calling and receiving orders from the simulated physicians. Radiology students and Physician Assistant students have been included to participate in this simulation experience. Students simulate receiving report and learning to communicate appropriately among interdisciplinary departments. Including different disciplinarians allows all students to view each profession and learn to interact professionally and appropriately.

The inter-professional simulation is a comprehensive simulation to allow students to demonstrate their ability to care of several patients with a variety of conditions. To meet the objectives, students must be able to appropriately recognize abnormal assessment findings and lab values. Utilizing their acquired critical thinking skills, students 
recognize the need to notify the physician, obtain orders and carry them out appropriately.

\section{Framework}

TeamSTEPPS is an evidence-based system to assist in improving communication and teamwork among healthcare professionals [5]. There are five key principles to TeamSTEPPS that help assist in the improvement of communication and team work. The five key principles are: Team structure, Communication, Leadership, Situation Monitoring, and Mutual Support [5](Figure 1). In order to have a good team structure, team members must learn to effectively communicate with one another, demonstrate their ability to lead, constantly be monitor their situation, and give members mutual support. There are several acronym techniques that TeamSTEPPS provide to assist faculty in developing better communication among students and teams. In the ADNP, the key principles are assigned through each semester to assist students in progressing to a welldeveloped team structure. In the final semester students continue to build upon what they have previously learned and demonstrate their ability to perform the four key principles to a team structure in the inter-professional simulation.

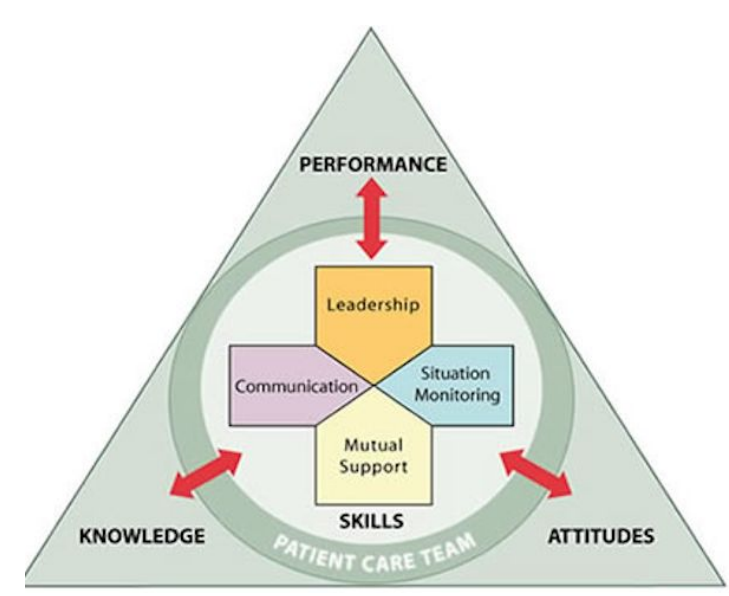

Figure $1:$ TeamSTEPPS

In each simulated activity, students are required to use the techniques and strategies within TeamSTEPPS. The most commonly used techniques used in the final semester are SBAR, Call-out, and Check-back. During the debriefing session, faculty use the debrief checklist in order to organize critical thinking skills while recognizing areas that need improvement [5]. Students use the technique SBAR when communication with simulated physicians to learn how to effectively communication patient information (Figure 2). The " $\mathrm{S}$ " is used to give the physician a situation that is occurring. The " $\mathrm{B}$ " is to give the physician a background of the patient to ensure correct identification. The " $A$ " is when students give their assessment findings to the physician, and the " $R$ " is for any recommendations the nurse feels is necessary. The SBAR technique is used in all situations and has recently been made the requirement for email communication between students and faculty. The Call-out strategy is used during our emergent simulations, such as ACLS. During the activity, students are required to "call-out" vital signs, assessment findings, medications given, and any other information necessary for the team to be knowledgeable about. The Check-back strategy is to allow students to become familiar with repeating and ensuring they have received correct information. The most common time this strategy is used is when the student receives orders from the simulated physician. The student should repeat back the orders to ensure accuracy, which increases patient safety.

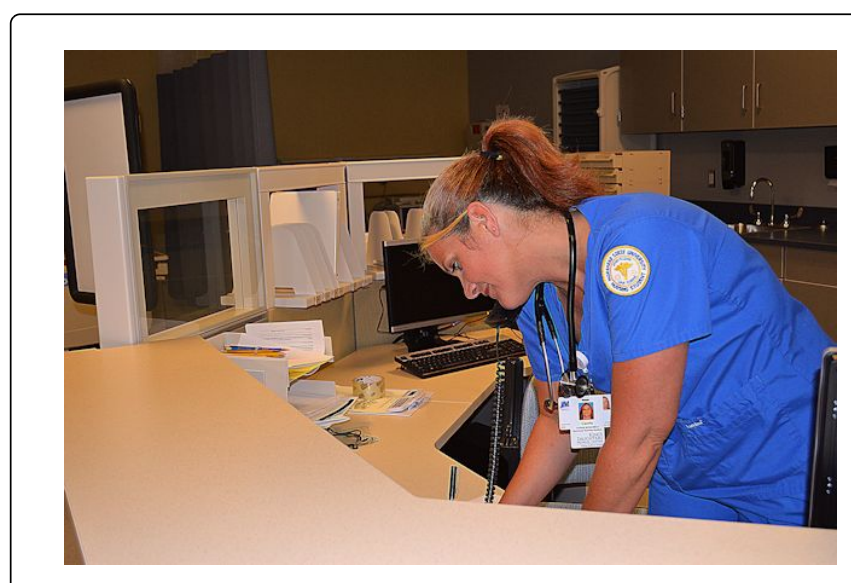

Figure 2: Students use SBAR when communicating with the simulated physician.

\section{Roles Assigned}

During each simulation lab, students participate in many different roles in order to facilitate understanding for delegation decisionmaking and recognizing specific duties that can be performed among different roles. For example, students should demonstrate the ability to delegate appropriate tasks to the nurse assistant versus the registered nurse. The student roles include: charge nurse, primary nurse, nurse assistant, family member, and the patient. In the charge nurse role, the student is evaluated on their ability to manage a situation and adequately serve as a leader during a simulated scenario. The primary nurse role is evaluated on the ability to adequately perform an assessment, determine the issue, and correctly communicate any needed information to the charge nurse. The nurse assistant role is evaluated on their ability to perform tasks specific to their role and not to practice outside of their scope of practice as written in state board of nursing regulatory guidelines. This particular role allows faculty to evaluate how students delegate in this role and to make sure tasks are appropriate for the nurse assistant role. The family member role allows students to recognize the impact our actions and communication can have on family members. The students are evaluated in two different areas within this role. First, the student acting in the family member role, views the situation from the eyes of loved ones at the bedside. They assist with evaluating faculty on how they were made to feel comfortable and kept informed regarding the situation. Second, faculty evaluates how students interact and communicate with family at the bedside. Exposing students to the various roles and allowing them to practice these roles during each simulation activity helps to prepare them for entering the healthcare field and being cognizant of different roles. Students gain confidence while learning to adapt to different roles and recognizing areas to improve during their clinical rotations (Figure 3). 


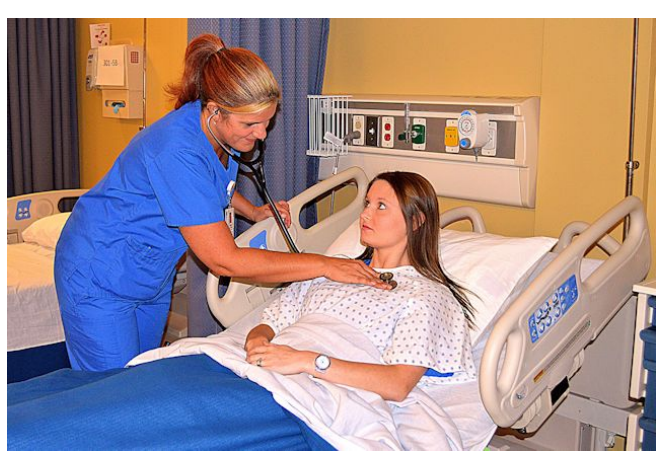

Figure 3: Student playing the role of patient.

\section{Preparing Students Prior to Simulation Labs}

By preparing students prior to the simulation activity, students will have a better understanding of what will be required and by doing this students will have a better learning experience. Prior to a simulation activity, students receive an assignment they complete prior to coming to lab. For example, prior to the ACLS simulation, the assignment includes specific medications and laboratory tests for students to define, cardiac rhythms that students create the appropriate algorithm and articles specific to the content for students to read in order to assist in the preparation of the lab experience. The assignments are titled ticket to simulation. Students present their assignments in order to complete the lab. Students also receive a role assignment sheet that lists what roles will be used and role expectations. Students discuss among their selves and determine which student fits in the best role. This activity helps students to determine their strengths and weaknesses (Figure 4).

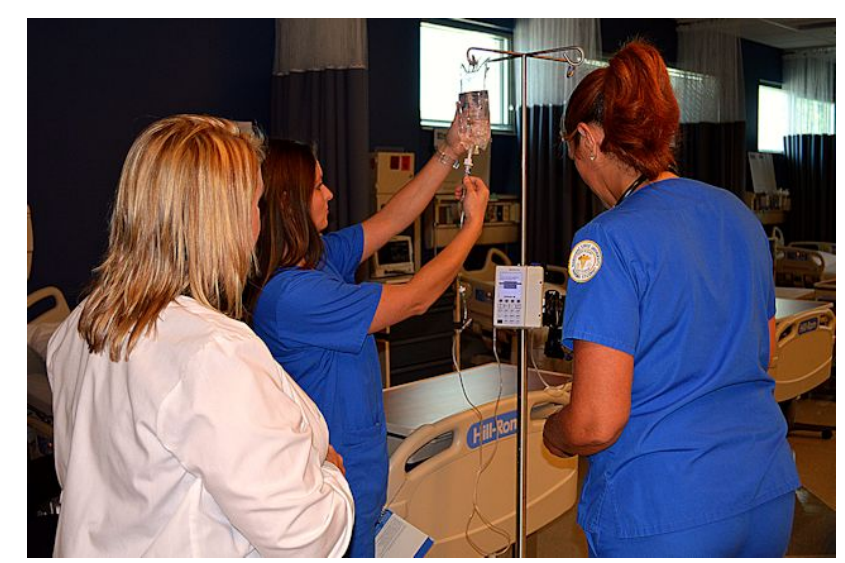

Figure 4: Students being oriented to equipment by faculty prior to simulation activity.

\section{Debriefing}

Debriefing is a period where students come together and discuss a simulated activities outcome. Debriefing after the simulation activity is a time for reflection for the students and reinforces the learning process. Students are encouraged to reflect and to recognize any areas of the simulation activity they would change or areas that need improvement. The debriefing session is an important component of the simulation activity and is facilitated by the nurse educator. It is important for educators to allow time for positive reinforcement and maintain the safe environment for student learning. If educators are too critical when discussing student mistakes, communication could be diminished and learning could be stifled. Maintaining psychological safety promotes a positive learning environment, which is necessary to encourage learning [6]. During the debriefing, students are encouraged to explain why certain tasks were performed and how they prioritized their tasks. This allows the educators to determine any confusion among didactic content and the actual skills performance. During this time students recognize and receive clarification for content not understood. Team STEPPS includes a debriefing checklist to assist faculty in the debriefing session [5]. Questions are selected and refined to be specific with each simulated activity. A few examples of questions used are: "What went well?, "Did team members understand their roles and responsibilities?", "What errors were made and how could they have been avoided?", and "What areas were recognized that improvement is needed?" (Figure 5).

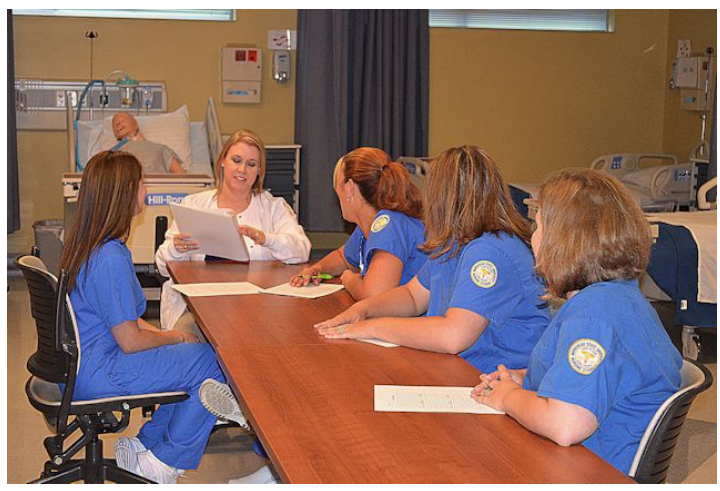

Figure 5: A debriefing session follows each simulation activity.

\section{Preparing Students for the Workforce}

While participating in simulation activities students recognize the areas of weakness that must be improved for successful transition into the workforce. Skills such as delegation, prioritization and communication assist students in preparing for the leadership role. Many will become charge nurses after passing the licensure exam. In addition, students learn to recognize certain symptoms that may indicate an emergent situation is imminent or possible. The ACLS simulation allows students to become familiar with an emergency situation. Graduate students have shared with the author that they were prepared when they encountered their first emergency situation. Learning skills prior to entering the workforce allows students to gain confidence to adequately perform which makes the transition from student to nurse easier.

\section{Simulation for Hospital-Based Nurses}

Simulation is a tool used in several careers to facilitate a safe learning environment. The military and airline pilot training programs use simulation to train personnel prior to engaging in combat or piloting an aircraft. Simulation is a useful tool for careers with ongoing learning, which is why it is so useful, not only in nursing education, 
Page 5 of 6

but in hospital settings as well. Simulation is implemented in several nursing programs to enhance student learning of skills and communication practice as well as in hospitals for practicing nurses. Simulation can be used to supplement orientation, facilitate transfer to a different practice unit, determine competency level of nurses, and enhance communication and safety.

Nursing is a career that requires continued growth and professional development with the implementation of new technologies and evidence-based care practices. The Institute of Medicine (IOM) recommends simulation to assist nurses in the ongoing development of knowledge and skills [7]. Some facilities are implementing simulation to assist in the orientation process or to learn new practices and skills. One hospital in Boston, Massachusetts piloted a simulation program and the program was so successful that they received funding to begin a simulation center that will "focus on nurses with varying levels of experience" [7]. The simulation programs include different settings such as, critical care and emergency care, with multiple scenarios in order to enhance the continued learning of practicing nurses [7]. There are several ways for simulation activities to be performed; role play, simulated scenarios, and/or unfolding case study simulations. Facility nurse educators can decide which type of simulation would be most beneficial for their nurses.

Simulation activities for practicing nurses could be utilized to enhance therapeutic communication and also enhance already in place safety measures. Incorrect communication can lead to serious safety issues among practicing healthcare professionals. By implementing simulation activities, nurses and other healthcare professionals simulate communication using evidence-based communication skills, such as, SBAR. By simulating in the scenario, practicing nurses and other healthcare professional can improve their communication skills and learn to coordinate as a team [8].

Simulation could also be used as an evaluation tool for facilities to determine their employee's competency level. As new employees are hired, their competency level determined from a simulation activity could determine what specific areas they are eligible for employment. Activities could include role-play and/or the use of manikins with varying fidelity to determine their level of competency. Simulation practice could enhance one's learning to assist them to become competent in specific critical areas. Simulation is an effective learning tool to enhance the performance of practicing nurses in numerous situations $[7,8]$.

\section{Evaluation of Student Simulation Experience}

In order to evaluate the effectiveness of teaching using the simulation experience, quantitative data is collected at the end of each semester. Faculty review the data and implement changes where indicated in the simulation assignments. Quantitative analyses from the last four semesters demonstrate effectiveness of simulation in four specific areas. The specific areas include meeting the course student outcomes, incorporating management skills into patient care, incorporating safety into clinical practice, and improving clinical decision making. Table 1 is a review of the quantitative data from the last four semesters.

\begin{tabular}{|l|l|l|l|l|l|}
\hline Indicators & Spring 2014 & Fall 2013 & Spring 2013 & Fall 2012 & $\begin{array}{l}\text { Average Total for each } \\
\text { indicator }\end{array}$ \\
\cline { 2 - 6 } & $\mathbf{N}=\mathbf{2 8}$ & $\mathbf{N}=\mathbf{3 0}$ & $\mathbf{N}=\mathbf{2 5}$ & $\mathbf{N}=\mathbf{2 4}$ & \\
\hline $\begin{array}{l}\text { Lab experiences (skills/simulation) were adequate in } \\
\text { meeting the course student outcomes. }\end{array}$ & $89.30 \%$ & $93.33 \%$ & $80 \%$ & $95.80 \%$ & $90 \%$ \\
\hline $\begin{array}{l}\text { The simulation helped me to incorporate management skills } \\
\text { into patient care (prioritization, coordination, collaboration, } \\
\text { delegation, effective utilization of resources). }\end{array}$ & $85.70 \%$ & $90 \%$ & $88 \%$ & $83.30 \%$ & $87 \%$ \\
\hline $\begin{array}{l}\text { The simulation helped me to incorporate patient safety } \\
\text { issues into my clinical practice. }\end{array}$ & $89.30 \%$ & $96.70 \%$ & $84 \%$ & $95.80 \%$ & $91 \%$ \\
\hline $\begin{array}{l}\text { Participation in the simulation helped me improve my } \\
\text { clinical decision making. }\end{array}$ & $89.30 \%$ & $86.70 \%$ & $80 \%$ & $87.50 \%$ & $86 \%$ \\
\hline Average Totals per Semester & $88.40 \%$ & $91.70 \%$ & $83 \%$ & $91 \%$ & $88.53 \%$ \\
\hline
\end{tabular}

Table 1: Review of the quantitative data from the last four semesters

\section{Conclusion}

Upon review of quantitative data, the analysis demonstrates the necessity for simulation in nursing education. Simulation is an important element in nursing education. Students practice skills and acquire an understanding of content taught in the didactic setting. Once students have a better understanding of content learned, they are better able to transfer theory into practice. Enhancing the performance of students in the clinical setting allows them to gain the confidence needed to perform professionally. Eight-seven percent of students agree that simulation assists in their knowledge of management skills. Management skills are necessary for nurses to prioritize and collaborate in order to perform adequate patient care. Eighty-six percent of students agree that simulation improves their clinical decision making skills. Clinical decision making skills are necessary to assist nurses in making appropriate decisions with their patient care. Ninety-one percent of students agree that simulation teaches them to incorporate safety in their clinical practice. Nurses must always keep their patients safe during their care. With the advancing technology and new evidence-based learning strategies, it is important for educators to implement evidence-based simulation research and enhanced technologies to promote learning for nurses. 


\section{References}

1. Nehring WM, Lashley FR (2009) Nursing simulation: A review of the past 40 years Simulation \& Gaming, 40: 528-552.

2. Lasater K (2007) High-fidelity simulation and the development of clinical judgment: Students' experiences. Journal of Nursing Education, 46 269-275.

3. Wane D, Lotz K (2013) The simulation clinical environment as platform for refining critical thinking in nursing students: A pilot program. Nursing Education Perspectives, 34: 163-166.

4. Tawalbeh L, Tubaishat A (2014) Effect of simulation on knowledge of advanced cardiac life support, knowledge retention, and confidence of nursing students of Jordan. Journal of Nursing Education, 53: 38-44.
5. AHRQ (2014). TeamSTEPPS. Agency for Healthcare Research and Quality.

6. Chesak SS, Dreifuerst KT(2010) Letters to the EDITOR. Nursing Education Perspectives,31: 46.

7. Aebersold M, Tschannen D (2013) Simulation in nursing practice: The impact on patient care. The Online Journal of Issues in Nursing, 18

8. Kilpfel JM, Carolan BJ, Brytowksi N, Mitchell CA, Gettman MT et al. (2014). Patient safety improvement through in situ simulation interdisciplinary team training. Urologic Nursing, 34: 39-46. 